\title{
Category Deficits and Paradoxical Dissociations in Alzheimer's Disease and Herpes Simplex Encephalitis
}

\author{
Keith R. Laws ${ }^{1 *}$ and Giuseppe Sartori ${ }^{2}$
}

\begin{abstract}
Most studies examining category specificity are single-case studies of patients with living or nonliving deficits. Nevertheless, no explicit or agreed criteria exist for establishing category-specific deficits in single cases regarding the type of analyses, whether to compare with healthy controls, the number of tasks, or the type of tasks. We examined two groups of patients with neurological pathology frequently accompanied by impaired semantic memory (19 patients with Alzheimer's disease and 15 with Herpes Simplex Encephalitis). Category knowledge was examined using three tasks (picture naming, naming-to-description, and feature verification). Both
\end{abstract}

\section{INTRODUCTION}

Although various tasks and statistical techniques have been used to try to document category effects, a critical and surprisingly unaddressed question concerns what would be considered acceptable as evidence of a "category effect" (Laws, 2004, in press). Most category effects reflect an initial demonstration of poor picture naming for one category and, in some cases, poor picture naming is the only evidence presented (e.g., in studies of Alzheimer's patients, see Laws, in press). One thing is certain, that is, that picture naming is implicitly viewed as the main task for confirming the presence of a category effect (and then that the additional tasks are used to elaborate on that). This assumption reflects the fact that most models predict that naming is impaired if semantic memory is impaired (and vice-versa). Nevertheless, evidence has existed for some time to show that this is not necessarily the case. For example, Laws, Evans, Hodges, and McCarthy (1995) described patient SE who had impaired (associative, nonvisual) semantics for animals but could name pictures of animals.

Recent studies have raised issues about the statistical methods used to assess category effects with naming data (Laws, in press; Laws, Gale, Leeson, \& Crawford, 2005). In particular, the common practice of making comparisons between the absolute number of living and

\footnotetext{
${ }^{1}$ Nottingham Trent University, UK, ${ }^{2}$ University of Padua, Italy *Now at the University of Hertfordshire, UK
}

patient groups were compared with age- and educationmatched healthy controls. The profile in each patient was examined for consistency across tasks and across different analyses; however, both proved to be inconsistent. One striking finding was the presence of paradoxical dissociations (i.e., patients who were impaired for living things on one task and nonliving things on another task). The findings have significant implications for how we determine category effects and, more generally, for the methods used to document double dissociations across individual cases in this literature.

nonliving items that are named (verified, identified, and so on) rather than comparing the patient data with control data. This may be misleading about the presence and even direction of category effects. Laws (in press) noted several consequences of failing to evaluate patient naming against the performance of matched normal healthy controls. The most common analysis in over two-thirds of all category-specific studies is the withinpatient comparison of the absolute scores (using $\chi^{2}$ : Laws, in press); and this reveals quite different dissociations from those disclosed when a control comparison is included. Indeed, Laws, Gale, et al. (2005) have demonstrated how patients may show false negatives, false positives, and even paradoxical dissociations (i.e., impaired for living on one task and for nonliving on another task). Hence, the absolute living-nonliving naming difference alone (be it exceptionally large or nonexistent) provides an unreliable indicator for both the presence and the direction of category effects.

This raises an important related methodological issue concerning how we define a category effects through a test profile. In fact, no a priori test profile exists for documenting a category disorder (e.g., a category difference for picture naming, attribute verification, naming-to-description, fluency, drawing or any combination of these). It also raises a critical issue concerning whether such disorders are empirically and/or theoretically driven. For example, we could make an arbitrary empirical decision such that for a category effect to be convincing, minimally requires a patient 
to show the same significant category effect on three tasks: A (picture naming), B (drawing), and C (attribute verification). Nevertheless, what if another patient is impaired on A and B, but not C? This patient may still have a category disorder and tell us something potentially more important about the true nature of category effects (e.g., that they might be related to visual knowledge alone).

Finally, although the vast majority of category-specific studies use a case study approach (for a review, see Laws, in press), a minority have used between-group comparisons. One reason that group studies have been less popular in this area is because group analyses may cancel out individual category effects. For example, if some patients showed a living deficit and some a nonliving deficit, then the overall pattern might simply reveal poor performance on both (see Gonnerman, Anderson, Devlin, Kempler, \& Seidenberg, 1997). This is especially likely with severely neurologically impaired cases. The following experiments compare category knowledge on three tasks in healthy controls with patients with Alzheimer's disease and Herpes Simplex Encephalitis (HSE); and are designed to explore how category effects might be determined.

\section{EXPERIMENT 1: THE LIVING-NONLIVING DISCREPANCY IN ALZHEIMER'S DISEASE}

The incidence and pattern of category specificity across Alzheimer's patients as a group (Tippett, Grossman, \& Farah 1996; Silveri, Daniele, Guistolisi, \& Gainotti 1991) and for individual Alzheimer's patients (Laws, Leeson, \& Gale, 2003; Garrard, Patterson, Watson, \& Hodges, 1998; Gonnerman et al., 1997; Mauri, Daum, Satori, Riesch, \& Birbaumer, 1994) have been inconsistent.

Most analyses of Alzheimer's patients have reported living deficits, a minority has reported nonliving deficits, some report both, and still others find no category specificity in Alzheimer's patients (for a review, see Laws, Gale, et al., 2005). Some of this variability may stem from previously ignored methodological problems. In particular, Laws, Gale, et al. (2005) note that controls in these studies are often performing at ceiling level, and have shown how this may distort the incidence and even direction of category effects. An additional issue concerns the observation that almost all of the studies examining category effects in Alzheimer's patients have relied exclusively on picture naming as the test of category. The current study addresses these points by testing picture naming, naming-to-description, and feature verification in Alzheimer's patients and controls.

\section{Results}

The criterion for a living-nonliving dissociation was that a patient must show a discrepancy that would be estimated to occur in $<1 \%$ of the population of healthy matched controls. Two of the Alzheimer's patients were impaired on all three category tasks (see Table 1). Nine were impaired on picture naming ( 7 living and 2 nonliving), 10 on feature verification (all living), and 3 on naming to description (all living).

Critically, the analyses revealed category inconsistencies across tasks. One patient showed a paradoxical dissociation: patient LZ had a differential deficit for living things on the feature verification task, but for nonliving things on the picture naming task.

\section{Summary}

The comparison with results from $\chi^{2}$ points to a number of both false positives (7) and false negatives (5). In one patient (SL), $\chi^{2}$ produced significant dissociations on all three tasks, however, when referenced to control data,

Table 1. Category Performance in Alzheimer's Patients (\% Living and Nonliving)

\begin{tabular}{|c|c|c|c|}
\hline Patient & $\begin{array}{c}\text { Feature } \\
\text { Verification }\end{array}$ & $\begin{array}{c}\text { Picture } \\
\text { Naming }\end{array}$ & $\begin{array}{l}\text { Naming to } \\
\text { Description }\end{array}$ \\
\hline MS & $54 / 72 \mathrm{~L}^{\mathrm{s}} \chi^{2}$ & $15 / 47 \mathrm{~L}^{\mathrm{s} x^{2}}$ & $28 / 63 \mathrm{~L}^{\mathrm{C}} \chi^{2}$ \\
\hline $\mathrm{AE}$ & $48 / 66 \mathrm{~L}^{\mathrm{s}} x^{2}$ & $38 / 63 \mathrm{~L}^{\mathrm{s}} x^{2}$ & $25 / 58 \mathrm{~L}^{\mathrm{c}} x^{2}$ \\
\hline FR & $59 / 86 \mathrm{~L}^{x^{2}}$ & $41 / 66 \mathrm{~L}^{\mathrm{s}} \chi^{2}$ & $19 / 50 x^{x^{2}}$ \\
\hline $\mathrm{RN}$ & $53 / 69 \mathrm{~L}^{\mathrm{s}} \chi^{2}$ & $41 / 66 \mathrm{~L}^{\mathrm{s}} x^{2}$ & $28 / 54 x^{2}$ \\
\hline MR & $58 / 77 \mathrm{~L}^{\mathrm{s}} \chi^{2}$ & $22 / 47 \mathrm{~L}^{\mathrm{s}} \chi^{2}$ & $19 / 46 x^{2}$ \\
\hline VP & $21 / 38 \mathrm{~L}^{\mathrm{s}} x^{2}$ & $47 / 72 \mathrm{~L}^{\mathrm{c}} \chi^{2}$ & $6 / 33^{x^{2}}$ \\
\hline $\mathrm{LZ}$ & $49 / 61 \mathrm{~L}^{\mathrm{s}}$ & $72 / 53 \mathrm{NL}^{\mathrm{C}}$ & $47 / 63$ \\
\hline BP & $60 / 63$ & $32 / 53 \mathrm{~L}^{\mathrm{s}}$ & $34 / 38$ \\
\hline FB & $60 / 77 \mathrm{~L}^{\mathrm{c}} \chi^{2}$ & $47 / 47$ & $34 / 67 \mathrm{~L}^{\mathrm{c}} x^{2}$ \\
\hline $\mathrm{BA}$ & $78 / 77$ & $75 / 59$ & $34 / 63$ \\
\hline SL & $63 / 45^{x^{2}}$ & $91 / 66 x^{2}$ & $56 / 29 x^{2}$ \\
\hline MF & $58 / 77 \mathrm{~L}^{\mathrm{s} \chi^{2}}$ & $44 / 50$ & $28 / 46$ \\
\hline OP & $56 / 69 \mathrm{~L}^{\mathrm{s}}$ & $44 / 53$ & $22 / 29$ \\
\hline $\mathrm{AB}$ & - & $78 / 56 \mathrm{NL}^{\mathrm{c}}$ & - \\
\hline DS & - & $72 / 63$ & $53 / 50$ \\
\hline $\mathrm{RR}$ & - & $47 / 47$ & - \\
\hline $\mathrm{CC}$ & - & $13 / 25$ & - \\
\hline $\mathrm{VC}$ & $75 / 69$ & $44 / 41$ & $47 / 67$ \\
\hline HT & $83 / 88$ & $72 / 75$ & $78 / 63$ \\
\hline $\begin{array}{l}\text { Living/nonliving } \\
\text { control } \\
\text { range }(\%)^{*}\end{array}$ & 15.6 to -9.4 & 5.9 to -10.94 & 20.8 to -15.6 \\
\hline
\end{tabular}

$* \mathrm{~L}=$ differential living deficit; $\mathrm{NL}=$ differential nonliving deficit; $\chi^{2}=$ patient impaired using $\chi^{2} ; s=$ strong dissociation; $\mathrm{c}=$ classical dissociation.

$+=$ living advantage; $-=$ nonliving advantage 
none emerged as significant. Using the criterion of category-consistent dissociations across all three tasks, two patients (MS and AE) showed living impairments.

A further six patients were impaired on two tasks; critically, in patient LZ, the deficits were paradoxical, that is, impaired for living on one task (feature verification) and nonliving (picture naming) on another task. The case of LZ is of particular importance. Given that the features used in the features verification task were all perceptual/visual, and given that semantic activation from pictures is triggered from visual features of the picture itself, this paradoxical dissociation is particularly surprising. The lack of consistency across analyses and the finding of a paradoxical dissociation across tasks emphasize why performance on any one task cannot provide a reliable indicator of the direction of a category effect.

\section{EXPERIMENT 2: THE LIVING-NONLIVING DISCREPANCY IN HERPES SIMPLEX ENCEPHALITIS}

In contrast to the studies of category effects in groups of Alzheimer's patients, many of the reported case studies of patients with category-specific disorders are people who have suffered from HSE (22/47 living cases, and not one nonliving case; see anatomical review by Gainotti, 2000). The original report of category effects was in a series of four HSE patients (Warrington \& Shallice, 1984). As Gainotti (2000) argues, when referring to HSE, that "the selective impairment of Living things, far from being an occasional phenomenon resulting from idiosyncratic factors, is strongly associated with a certain kind of brain pathology."

Following the initial reports of Warrington and Shallice (1984), other patients suffering from the same pathology who also displayed living thing deficits increased confidence in the claim that "real" category specificity can be found predominantly in this group of patients. Capitani, Laiacona, Mahon, and Caramazza (2003) reported, in their database, 26/61 patients with HSE in the group impaired for living and 1/18 in that impaired for nonliving. Nevertheless, these figures refer to the incidence of HSE category-specific patients in the total population of category-specific patients. No data are available regarding the incidence of category specificity among the HSE population. For this reason, the second part of this study examines the incidence and type of category effects that occur in a series of patients following HSE.

\section{Results}

The data were analyzed using the same methods outlined in Experiment 1. One HSE patient was impaired across all three tasks (SR). Five patients were impaired on two of the three tasks. Two HSE patients (DD and MF) also displayed paradoxical dissociations (see Table 2).

\section{Summary}

Again, a large number of false negatives occur when using $\chi^{2}$ (11 for HSE patients). As with Alzheimer's patients, an absolute (nonreferenced) comparison is likely to underestimate rather than overestimate the incidence of category dissociations. Inconsistency across tasks for an individual patient again highlights the fact that performance on any one task cannot provide a reliable marker for a category effect-this includes feature verification (which was the most sensitive and reliable task in both groups for detecting significant category differences).

Furthermore, the analysis of HSE patients accentuates how absolute scores can be misleading. Consider BM, who showed a significant differential deficit for living things on feature verification in the $\chi^{2}$ analysis (76\% vs. $90 \%$ ), but not when compared to controls (although the

Table 2. Category Performance in HSE Patients (\% Living and \% Nonliving)

\begin{tabular}{|c|c|c|c|}
\hline Patient & $\begin{array}{c}\text { Feature } \\
\text { Verification }\end{array}$ & $\begin{array}{c}\text { Picture } \\
\text { Naming }\end{array}$ & $\begin{array}{c}\text { Naming to } \\
\text { Description }\end{array}$ \\
\hline SR & $68 / 78 \mathrm{~L}^{\mathrm{s}}$ & $56 / 78 \mathrm{~L}^{\mathrm{s}}$ & $41 / 71 \mathrm{~L}^{\mathrm{s}} \chi^{2}$ \\
\hline SG & $76 / 78 \mathrm{~L}^{\mathrm{s}}$ & $75 / 94 \mathrm{~L}^{\mathrm{c} x^{2}}$ & $56 / 54$ \\
\hline MF & $79 / 86 \mathrm{~L}^{\mathrm{c}}$ & $100 / 72 \mathrm{NL}^{\mathrm{c}}$ & $75 / 79$ \\
\hline $\mathrm{TL}$ & $60 / 79 \mathrm{~L}^{\mathrm{s} x^{2}}$ & $38 / 31$ & $16 / 50 \mathrm{~L}^{\mathrm{s}} \chi^{2}$ \\
\hline DD & $56 / 57 \mathrm{~L}^{\mathrm{s}}$ & $53 / 44$ & $56 / 25 \mathrm{NL}^{\mathrm{s}}$ \\
\hline NP & $53 / 78 \mathrm{~L}^{\mathrm{s}} \chi^{2}$ & $66 / 59$ & $65 / 62$ \\
\hline$Z G$ & $90 / 82 \mathrm{~L}^{\mathrm{c}}$ & $97 / 91$ & $75 / 71$ \\
\hline GE & $90 / 86 \mathrm{~L}^{\mathrm{c}}$ & 94/94 & $81 / 67$ \\
\hline $\mathrm{BM}$ & $76 / 90^{x^{2}}$ & $75 / 94 \mathrm{~L}^{\mathrm{c} x^{2}}$ & $66 / 92 \mathrm{~L}^{\mathrm{c} x^{2}}$ \\
\hline $\mathrm{SO}$ & $82 / 89$ & $91 / 69 \mathrm{NL}^{\mathrm{c}}$ & $78 / 63$ \\
\hline $\mathrm{TF}$ & $81 / 89$ & $100 / 84 \mathrm{NL}^{\mathrm{c}}$ & $78 / 88$ \\
\hline RU & $85 / 94$ & $91 / 94$ & $84 / 79$ \\
\hline $\mathrm{LF}$ & $93 / 99$ & $94 / 97$ & $85 / 83$ \\
\hline $\mathrm{RF}$ & $94 / 97$ & $97 / 100$ & $88 / 79$ \\
\hline MA & $94 / 89$ & $97 / 91$ & $91 / 79$ \\
\hline $\begin{array}{l}\text { Living/nonliving } \\
\text { control } \\
\text { range }(\%)^{*}\end{array}$ & 15.6 to -3.1 & 0.6 to -7.8 & 20.8 to -9.4 \\
\hline
\end{tabular}

$* \mathrm{~L}=$ differential living deficit; $\mathrm{NL}=$ differential nonliving deficit; $\chi^{2}=$ patient impaired using $\chi^{2} ; s=$ strong dissociation; $\mathrm{c}=$ classical dissociation.

$+=$ living advantage $;-=$ nonliving advantage . 
difference was larger than 6 of 8 patients who showed a significant difference using the Revised Standardized Difference Test [RSDT]). The absolute size of the difference is therefore not a definitive marker for the presence or absence of a differential deficit. Moreover, consider patients GE (90 vs. 86\%) and ZG (90 vs. $82 \%$ ), who showed better absolute feature verification for living than for nonliving things, yet had differential living deficits (indeed, classical dissociations). So, not only do absolute differences provide no guarantee to the presence of a differential deficit, they provide no guarantee about the direction of any deficit.

\section{GENERAL DISCUSSION}

This study investigated the validity of the criteria that are commonly used to document the presence of categoryspecific deficits, in particular, the strong dependence upon within-patient comparisons of absolute living and nonliving scores rather than comparing patient performance with that of healthy controls. Additionally, most studies have relied upon establishing category disorders on picture-naming tasks; and in some studies (especially those examining Alzheimer's patients), rely solely upon picture naming as evidence of category specificity (Laws, Gale, et al., 2005). The current study illustrates how such methods lead to erroneous conclusions about both the presence and even the type of category deficit exhibited by patients. These findings have implications for a range of questions relating to category disorders and raise methodological and theoretical issues regarding what might be regarded as acceptable evidence for dissociations and double dissociations-especially when used to make claims about the fractionation of cognitive architecture.

\section{Use of Chi-square Analysis}

The current study highlights the unreliability of using within-patient comparison of absolute scores. As already mentioned, studies of Alzheimer's patients frequently assess picture naming only, and in line with the data presented here, would be likely to misrepresent not only the incidence, but even the direction of a category effect. The nature of $\chi^{2}$ analyses means, of course, that studies always assume the deficit to be reflected in the smaller of any two scores obtained. The current study shows how this metric is unreliable and confirms our recent findings in two other groups of Alzheimer's patients (Laws, Gale, et al., 2005).

The widespread use of within-patient comparisons to determine category deficits may well have distorted the incidence, and therefore, the ratio of living to nonliving deficits reported in the literature. Even if patients show a patently large absolute difference across category, this does not necessarily denote a category disorder; or conversely, does the failure to find a large absolute difference across category refute the presence of a category deficit. Indeed, several HSE and Alzheimer's patients showed large absolute differences that were significant using within-patient $\chi^{2}$ analyses, but not when referenced to control data (e.g., HSE patients such as BM, DD, and SG for picture naming; and Alzheimer's patients such as RN, MR, and VP for naming-todescription). Conversely and more commonly, $\chi^{2}$ analyses led to false negatives in both HSE (11 dissociations) and Alzheimer's (7 dissociations) patients. This reflects the simple fact that absolute differences are critical for demonstrating deficits when using $\chi^{2}$; however, healthy controls may show category advantages, and therefore, even a lack of absolute difference in patients may be important. For example, analysis of picture-naming for HSV patients DD and SG indicated no category difference ( 56 vs. $57 \%$ and 76 vs. $78 \%$ ), yet both showed significant dissociations when referenced to control data. Conversely, Alzheimer's patient FR showed a 31\% difference in living and nonliving naming-to-description (19 vs. 50\%), but this was not significant when referenced to control data.

\section{Picture Naming as Indicator of Semantic Impairment}

As outlined in the Introduction, the extant literature relies heavily upon using impaired picture naming to identify category effects; other tests may be used subsequently to elaborate on this, but picture naming has assumed a role as the sine qua non test of category specificity. Therefore, the failure to find a category difference on naming tasks is typically viewed as undermining the presence of any category effect (Laws, 1998; Laws, Evans, et al., 1995).

Although most cognitive psychologists are comfortable with the notion of impaired naming and intact semantics (i.e., lexical deficits), the converse-impaired semantics and intact naming-have been less frequently reported, perhaps because of the assumption that it ought not to happen within most currently accepted models (Laws, Evans, et al., 1995). Nonetheless, the current study documents several Alzheimer's (2/15: $13 \%)^{1}$ and HSE (3/15: 20\%) patients who showed a category effect in semantics (using feature verification), but no category effect on either of two naming tasks.

Some patients (2/19 Alzheimer's and 4/15 HSE) showed category deficits on tasks other than picture naming, including patients (e.g., Alzheimer's patient FB and 2 HSE patients DD and TL) with impaired performance on all tasks except picture naming. Therefore, using picture naming as a critical test of category effects would miss some patients who showed consistent deficits on other tasks. Indeed, reliance on any one task may lead to a quite different conclusion from that if another task had been chosen. Moreover, feature veri- 
fication proved to be a much more sensitive test for detecting category effects (albeit all living deficits) than picture naming or naming-to-description (although there were also differences across pathologies). This suggests that the direction of category effects sometimes seems to depend upon which test is chosen as the reference test (a prospect that has not been previously entertained). In this context, it is worth noting that patients who show living disorders tend to be agnosic, and therefore, tested with picture naming; however, several nonliving cases have been aphasic, and thus, were not tested with picture naming, but with tasks such as picture-name matching (see Laws, 2004). Hence, it is common for different category effects to rely upon different testing procedures; and as such, again the existing literature may be prone to some of the issues raised here.

\section{Dissociations and Paradoxical Dissociations}

Dissociations often form the basis for speculations about cognitive architecture and modularity especially when they are doubly dissociated between patients. The current study shows, however, that dissociations can occur within a patient. Within-patient double dissociations across tasks (e.g., a living on Task $\mathrm{A}$ and a nonliving on Task B) that are believed to have some critical processing stage in common, raise questions about the double-dissociation methodology in single-case studies and the interpretation of category effects per se. At a theoretical level, many models assume that deficits in semantics will have "knock-on" effects for naming; and so, such models have difficulty accounting for paradoxical dissociations at the level of semantics and naming.

Paradoxical double dissociations pose problems for double dissociations at a variety of levels of comparison including: across tasks (as described here), within tasks, and patients (Laws, Gale, et al., 2005); and of course, across patients and across tasks (the typical approach in category specificity and cognitive neuropsychology more generally). Given that paradoxical dissociations arise, how might we distinguish a paradoxical dissociation from a real double dissociation (i.e., one that might be used to ground theories of cognition or "carve cognition at its modular joints")?

How should paradoxical dissociations be interpreted? Of course, it might be argued that paradoxical dissociations are simply unreliable. Indeed, because we did not retest patients, we have no way of confirming whether paradoxical dissociations are reliable. Nonetheless, the reliability of paradoxical dissociations has to be viewed alongside the fact that reliability is hardly ever examined for dissociations in single-case studies. Indeed, follow-up analyses of the same patient by same or other research groups are rare and sometimes contradictory (Laws, 1998). Hence, it is crucial for future studies to examine the reliability of all dissociations.
We must also consider the possibility that paradoxical double dissociations reflect confounding variables. It might be argued, for example, that fluctuations in attention could impact differentially over the test session and potentially affect one category more than the other. This is unlikely because it would require that the confound interacts highly selectively with category. Living and nonliving stimuli (on all tests) were randomly intermixed when presented, so a factor such as attention fluctuation would have to impact only when items from one of the two categories were presented. This seems even more implausible in cases when we consider paradoxical dissociations (i.e., in the opposite direction on a second test). Consider the case of the HSE patient MF (see Figure 1), who showed a classical double dissociation across tasks. His picture naming was below the 1\% for living things (and normal for nonliving things); and below the 1\% for nonliving on feature verification (but normal for living things). In this context, we would argue that the dissociations are robust to any artifacts of this kind.

Another potential confound concerns the possibility that the dissociations reported here (whether consistent or paradoxical) are chance findings emerging from quite noisy patient data, and that multiple analyses might increase the likelihood of spurious outcomes. Indeed, typical statistical/methodological approaches may well be prone to producing spurious and chance findings in case study analyses. Nevertheless, Monte Carlo simula-

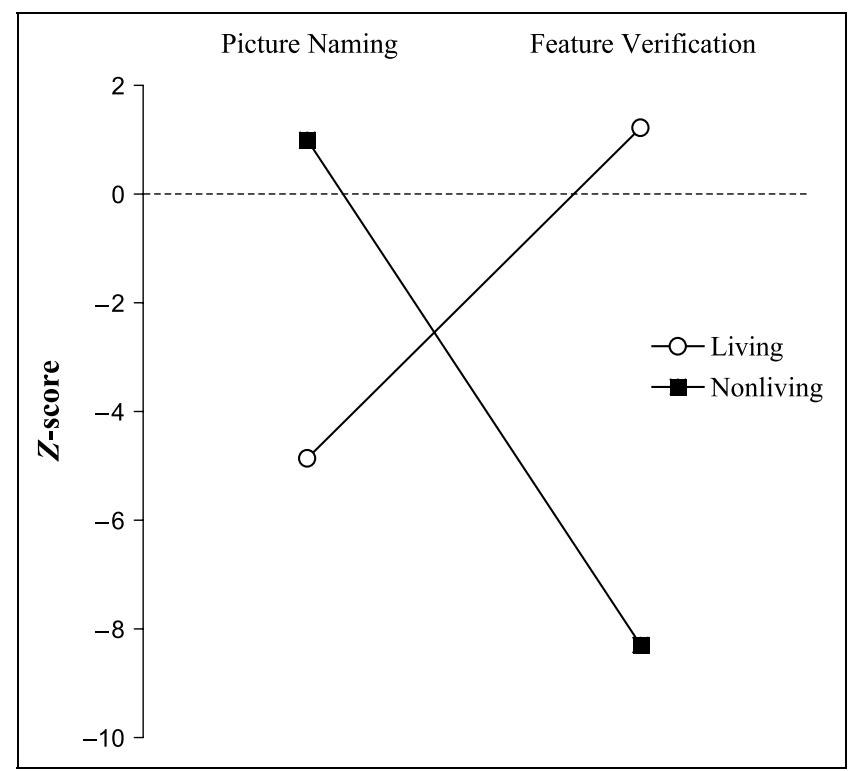

Figure 1. A classical paradoxical double dissociation between living and nonliving things within one HSE patient (MF). Note: Patient MF displays a classical double dissociation across category (i.e., impaired picture naming for living things, but normal nonliving thing naming). On feature verification, he shows normal living and impaired nonliving performance. Classical double dissociations (often with weaker evidence than here) typically provide the strongest evidence for the separation of cognitive processed (or architecture). 
tions show that the RSDT (Crawford \& Garthwaite, 2005) used in the current study provides excellent control over Type 1 error rate (even when patient data are heavily skewed through poor performance).

The dissociations reported here within one patient are indistinguishable from those typically reported between patients in the category-specific literature. Despite the equivalence of paradoxical and "regular" dissociations (and double dissociations) in terms of their acceptability as empirical evidence, it may be that both are unreliable. Hence, we should not take any one (or even some combinations) test as prima facie evidence of a category effect in one direction. As noted above, reliability, both across task and across time, are critical issues for future studies to consider in the case study approach.

\section{METHODS}

\section{Experiment 1}

\section{Participants}

The study included 19 (15 women and 4 men) patients with probable Alzheimer's dementia and 15 healthy elderly control subjects (9 women and 6 men). The 19 Alzheimer's patients met the National Institute of Neurological and Communicative Disorders and Stroke/Alzheimer's Disease and Related Disorders Association (NINCDS/ADRDA) criteria for probable Alzheimer's disease (McKhann et al., 1984). All 19 patients had Hachinski scores of less than 4 (Hachinski et al., 1975) and a Mini-Mental State Examination (MMSE) score below 24/30 (Folstein, Folstein, \& McHugh, 1975). All underwent CT or MRI scanning, together with a screening battery, to exclude treatable causes of dementia. Patients with major depression, a history of stroke or TIA, alcoholism, head injury, or major medical illnesses were excluded. Patients were recruited in three hospitals and in four nursing homes located in Veneto (Italy) and were an unselected series.

The Alzheimer's patients and controls were matched for age [mean $(S D)$ : 77.9 (8.7) vs. $75.33(4.2): F(1,33)=$ $1.02, p>.05]$ and years of education $[4.73(0.80)$ vs. 6.28 (4.76): $F(1,33)=1.5, p>.05]$. They did, of course, differ significantly in their MMSE [19.31 (2.45) vs. 27.4 (1.11): $F(1,33)=126, p<.001]$.

\section{Tasks}

1. Picture naming: 32 living (20 animals and 12 fruits and vegetables) and 32 nonliving items matched for frequency, familiarity, and visual complexity (Sartori, Job, \& Zago, 2002).

2. Feature verification: 80 living and 64 nonliving items (e.g., "a tiger has stripes"). All features were perceptual. The examinee is required to respond if the feature belongs to the concept or not (Sartori et al.,
2002). Half of the stimuli required a "yes" response and half required a "no" response.

3. Naming-to-description: Originally published by Lambon-Ralph, Howard, Nightingale, and Ellis (1998) as a feature verification task, it was adapted to Italian as a naming-to-description task. Sixteen animals and 12 objects were used. Each concept was described by a perceptual and a functional/associative description, thus yielding a total of 32 descriptions for living and 24 descriptions of nonliving.

\section{Experiment 2}

\section{Participants}

This experiment included 15 (7 women and 8 men) patients with HSE and 12 healthy control subjects (6 women and 6 men). Herpes Simplex patients were all recruited in three hospitals located in Veneto (Italy), were native speakers of Italian, and all satisfied the following criteria: all had polymerase-chain-reactionproven HSE; all showed pathologic changes at CT scan or MRI which were usually bilateral, in the medialtemporal and inferior frontal areas; all underwent treatment with Acyclovir; and all were examined between 13 and 46 months postonset.

The HSE patients and controls were matched for mean $(S D)$ age $[53.66(14.77)$ vs. $54.47(12.63): F(1,25)=$ $0.22, p=.88]$ and mean $(S D)$ years of education $[7.73$ (3.17) vs. 10.41 (4.31): $F(1,25)=3.47, p>.05]$. They did differ significantly in their mean $[S D]$ MMSE scores [24.66 (3.26) vs. 28.68 (0.72): $F(1,25)=17.31, p<.001]$.

\section{Tasks}

The same three tasks used for the Alzheimer's patient were administered to the HSE patients.

\section{Methods of Analysis Used to Establish Category Specificity}

The data for each individual participant were examined by comparing performance with their respective control group using the RSDT (Crawford \& Garthwaite, 2005) for testing for deficits and dissociations in single-case studies. Of course, it is possible for patients to be impaired at naming living or nonliving things, but that the difference between their scores does not reach significance; equally, a patient may be severely impaired on both tasks, but still show differential impairment. This method examines whether the discrepancy observed for the patient is significantly different from the discrepancies observed for controls and provides a point estimate of the abnormality of the individual's discrepancy (i.e., it estimates the percentage of the population that would obtain a more extreme discrepancy). Patient performance was classified as impaired if the discrepancy score 
was estimated to occur in less than $1 \%$ of the healthy control population (two-tailed).

Patients were classified as displaying strong dissociations if they were (a) impaired at both tasks and (b) showed a significant discrepancy across category; and classified as displaying a classical dissociation if they were (a) impaired in one category, but performed normally on the other and (b) showed a significant discrepancy between the two categories (see Crawford \& Garthwaite, 2005; Crawford, Garthwaite, \& Gray, 2003).

These methods of testing for deficits and for differences (i.e., dissociations) are to be preferred over the use of $z$ and $z_{\mathrm{D}}$ as they treat the statistics of the control sample as statistics rather than as population parameters. Moreover, Monte Carlo simulations show that the RSDT (Crawford \& Garthwaite, 2005) provides excellent control over Type 1 error rate (regardless of whether the data are heavily skewed, the control sample is small in size, or the correlation between tasks). We contrasted results from application of this method with those derived from applying, on the same data, those derived from using $\chi^{2}$-the method most frequently used to evaluate discrepancies in living/nonliving accuracy in single cases (Laws, in press). Programs to run the RSDT analyses can be downloaded from www.abdn.ac.uk/ \%7Epsy086/dept/psychom.htm.

\section{Acknowledgments}

We thank Dr. Ivry and the reviewers for insights and helpful comments on an earlier version of this article.

Reprint requests should be sent to Dr. Keith R. Laws, School of Psychology, University of Hertfordshire, College Lane, Hatfield, Hertfordshire AL10 9AB, UK, or via e-mail: keith.laws@ ntu.ac.uk.

\section{Note}

1. Four dementia of Alzheimer's type patients were not tested on this task.

\section{REFERENCES}

Capitani, E., Laiacona, M., Mahon, B., \& Caramazza, A. (2003). What are the facts of semantic category-specific deficits? A critical review of the clinical evidence. Cognitive Neuropsychology, 20, 213-261.

Crawford, J. R., \& Garthwaite, P. H. (2005). Testing for suspected impairments and dissociations in single-case studies in neuropsychology: Evaluation of alternatives using Monte Carlo simulations and revised tests for dissociations. Neuropsychology, 19, 318-331.

Crawford, J. R., Garthwaite, P. H., \& Gray, C. D. (2003). Wanted: Fully operational definitions of dissociations in single-case studies. Cortex, 39, 357-370.

Folstein, M. F., Folstein, S. E., \& McHugh, P. R. (1975). "Mini-mental state": A practical method for grading the cognitive state of patients for the clinician. Journal of Psychiatric Research, 12, 189-198.

Gainotti, G. (2000). What the locus of brain lesion tells us about the nature of the cognitive defect underlying category-specific disorders: A review. Cortex, 36, 539-559.

Garrard, P., Patterson, K., Watson, P. C., \& Hodges, J. R. (1998). Category specific semantic loss in dementia of Alzheimer's type. Functional-anatomical correlations from cross-sectional analyses. Brain, 121, 633-646.

Gonnerman, L. M., Anderson, E. S., Devlin, J. T., Kempler, D., \& Seidenberg, M. S. (1997). Double dissociation of semantic categories in Alzheimer's disease. Brain and Language, 57, 254-279.

Hachinski, V. C., Iliff, L. D., Zihka, E., Duboulay, G. H., McAllister, V. L., Marshall, J., Russell, R. W. R., \& Symon, L. (1975). Cerebral blood flow in dementia. Archives of Neurology, 32, 632-637.

Lambon Ralph, M. A., Howard, D., Nightingale, G., \& Ellis, A. W. (1998). Are living and non-living category-specific deficits causally linked to impaired perceptual or associative knowledge? Evidence from a category-specific double dissociation. Neurocase, 4 , 311-338.

Laws, K. R. (in press). "Illusions of Normality": A methodological review of category-specific naming. Cortex.

Laws, K. R. (1998). A leopard never changes its spots. Cognitive Neuropsychology, 15, 467-479.

Laws, K. R. (2004). Strange and neglected domains in category specificity: The "normal" and the "nonliving". History and Philosophy of Psychology, 6, 19-39.

Laws, K. R., Evans, J. J., Hodges, J. R., \& McCarthy, R. A. (1995). Naming without knowing and appearance without associations-Evidence for constructive processes in semantic memory. Memory, 3, 409-433.

Laws, K. R., Gale, T. M., Leeson, V. C., \& Crawford, J. R. (2005). When is category specific in dementia of Alzheimer's type? Cortex, 41, 452-463.

Laws, K. R, Leeson, V. C., \& Gale, T. M. (2003). Inflated and contradictory category naming deficits in Alzheimer's disease? Brain and Cognition, 53, 232-234.

McKhann, G., Drachman, D., Folstein, M., Katzman, R., Price, D., \& Stadlan, E. M. (1984). Clinical diagnosis of Alzheimer disease: Report of the NINCDS-ADRDA work group. Neurology, 34, 939-944.

Mauri, A., Daum, I., Sartori, G., Riesch, G., \& Birbaumer, N. (1994). Category-specific semantic impairment in

Alzheimer's disease and temporal lobe dysfunction: A comparative study. Journal of Clinical and Experimental Neuropsychology, 16, 689-701.

Sartori, G., Job, R., \& Zago, S. (2002). A case of domain-specific semantic deficits. In E. M. E. Forde \& G. W. Humphreys (Eds.), Category specificity in brain and mind (pp. 25-49). Hove, Sussex: Psychology Press.

Silveri, M. C., Daniele, A., Giustolisi, L., \& Gainotti, G. (1991). Dissociation between knowledge of living and non-living things in dementia of the Alzheimer's type. Neurology, 41, 545-546.

Tippett, L. J., Grossman, M., \& Farah, M. J. (1996). The semantic memory impairment of Alzheimer's disease: Category specific? Cortex, 32, 143-153.

Warrington, E. K., \& Shallice, T. (1984). Category specific semantic impairments. Brain, 107, 829-854. 